\title{
IL-4 regulates chemokine production induced by TNF- $\alpha$ in keratocytes and corneal epithelial cells
}

Yoji Takano, Kazumi Fukagawa, Shigeto Shimmura, Kazuo Tsubota, Yoshihisa Oguchi, Hirohisa Saito

\begin{abstract}
Background-Eosinophils are thought to play a major role in the pathogenesis of corneal lesions in ocular allergies. The regulation of chemokine production in corneal cells by the Th2 cytokine, interleukin 4 (IL-4), was examined in order to investigate its role in ocular allergies.

Methods-Pure cultures of human corneal epithelial cells and keratocytes were exposed to tumour necrosis factor $\alpha$ (TNF- $\alpha$ ) and/or IL-4. 24 hours after exposure, culture supernatants were removed and concentrations of IL-8 and RANTES were quantified by ELISA assay.

Results-Simultaneous addition of IL-4 inhibited TNF- $\alpha$ induced IL-8 production in both corneal epithelial cells and keratocytes. TNF- $\alpha$ and IL-4 synergistically stimulated the production of RANTES in keratocytes.

Conclusion-Differential regulation of chemokine production from corneal cells by IL-4 may play a role in the selective recruitment of predominantly eosinophils to the ocular surface in ocular allergies. (Br f Ophthalmol 1999;83:1074-1076)
\end{abstract}

In the acute phase of severe ocular allergies such as vernal keratoconjunctivitis (VKC) and atopic keratoconjunctivitis (AKC), corneal lesions such as ulcers and erosions are often observed. Such lesions not only cause severe pain but are also potentially vision threatening complications that must be addressed by the clinician. Eosinophil major basic protein (MBP) has been detected in corneal plaque in $\mathrm{VKC}^{1}$ and is reported to inhibit corneal epithelial migration, ${ }^{2}$ suggesting that eosinophils may play an important role in the pathogenesis of corneal lesions in ocular allergies. Eosinophil infiltration is prominent during the late phase of allergic inflammation; however, the precise mechanisms of such selective recruitment are still not fully understood.

Interleukin 8 (IL-8) is a CXC $(\alpha)$-chemokine with strong selective chemoattractive properties against neutrophils, and RANTES is a $\mathrm{CC}(\beta)$-chemokine that is strongly chemotactic for eosinophils. ${ }^{3}$ One of the factors that may lead to the predominance of eosinophils at sites of allergic inflammation is the relative amounts of the chemokines, IL-8 and RANTES, present at such sites.

IL-4 is a cytokine produced by Th2 type lymphocytes and is known to play a key role in allergic inflammation. As elevated levels of IL- 4 have been observed in tears of patients with ocular allergies, ${ }^{4}$ we hypothesised that IL-4 in tears may regulate IL- 8 and RANTES production from keratocytes and corneal epithelium and contribute to the selective recruitment of eosinophils to the ocular surface.

\section{Materials and methods}

ESTABLISHMENT OF PRIMARY CULTURES

Human corneas were obtained from the Eye Bank Association of America and cultures were established by the method previously described by Cubitt et al. ${ }^{5}$ At second passage, cells were removed from the culture dishes by incubation with $1 / 10$ diluted $0.05 \%$ trypsin- $0.53 \mathrm{mM}$ EDTA (Gibco BRL, Grands Island, NY, USA) in phosphate buffered saline (PBS) for 5 minutes. Corneal epithelial cells were resuspended at a concentration of $3 \times 10^{5}$ cells $/ \mathrm{ml}$ with fetal bovine serum (FBS) free RCBM medium (Kurabo Co, Tokyo, Japan) and $100 \mu \mathrm{l}$ of the cell suspension was then preincubated in a 96 well culture plate (Becton Dickinson Labware, Lincoln Park, NJ, USA). Keratocytes were resuspended at a concentration of $3 \times 10^{5}$ cells $/ \mathrm{ml}$ with Dulbecco's modified Eagle's medium (DMEM, Gibco BRL) containing $15 \%$ FBS, and $100 \mu \mathrm{l}$ of the cell suspension was then preincubated overnight in a 96 well culture plate. After washing with PBS, the medium was changed to DMEM without FBS 24 hours before the induction experiments. Characterisation of each cell type was assessed by cell morphology and the differential staining to anticytokeratin antibodies. The cells derived from the corneal epithelial layer were almost entirely composed of polygonal cells characteristic of stratified squamous epithelium and cells derived from the stromal cells were devoid of such polygonal cells and consisted of spindle-shaped cells characteristic of fibroblasts. Ninety eight per cent of corneal epithelial cells stained positively with antikeratin AE1/AE3 (Progen Biotechnik GMBH, Heidelberg, Germany), while $100 \%$ of keratocytes stained positively with anti-vimentin (Boehringer Mannheim, Indianapolis, IN, USA). Primary cultures of corneal epithelial cells and keratocytes from three donor corneas were established.

\section{STIMULATION OF CELL CULTURES}

The cells were stimulated by TNF- $\alpha$ and/or IL-4 for 24 hours as previously reported. ${ }^{6}$ Tumour necrosis factor $\alpha(\mathrm{TNF}-\alpha)$, a proinflammatory cytokine, was used to stimulate the corneal cells as it has been reported to induce RANTES production from conjunctival epithelium ${ }^{6}$ as well as from keratocytes. In 

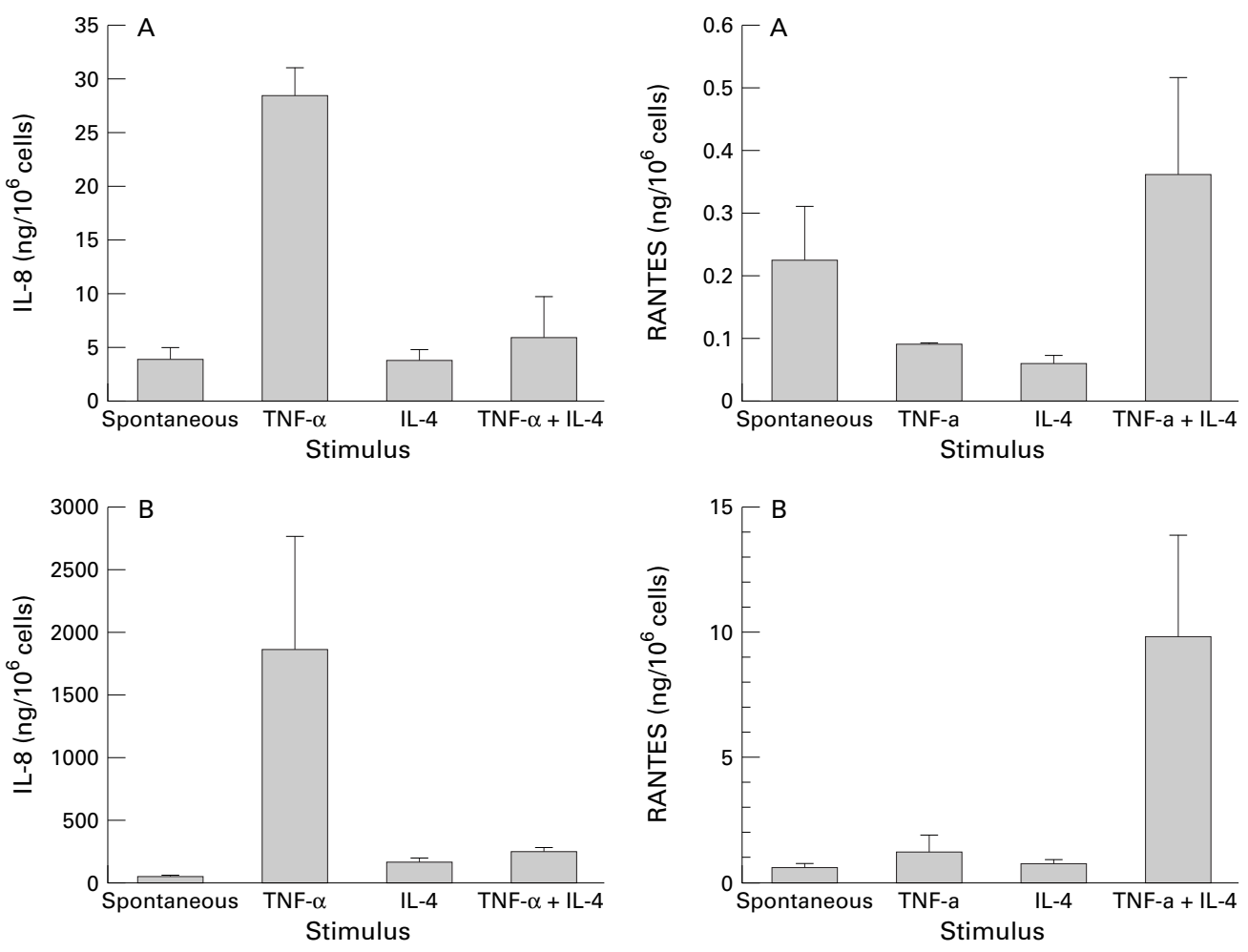

Figure $1 \quad$ IL-8 production by corneal epithelial cells $(A)$ and keratocytes $(B)(n=3$ in triplicate). Cells were stimulated with TNF- $a, I L-4$, and $T N F-a$ and $I L-4$ simultaneously. IL-8 was measured in 24 hour cell supernatants by ELISA. In the corneal epithelium, TNF-a induced IL-8 production ( $p=0.0001$ compared with spontaneous release). Simultaneous stimulation with $T N F-a$ and $I L-4$ resulted in the inhibition of $T N F_{-} \alpha$ induced IL-8 production ( $p=0.0002)$. Stimulation with $I L-4$ alone produced no significant effect on IL-8 production. In keratocytes, TNF- $a$ induced IL-8 production ( $p=0.025$ compared with spontaneous release). Simultaneous stimulation with $T N F-a$ and $I L-4$ resulted in the inhibition of $T N F-a$ induced $I L-8$ production $(p=0.039)$. Stimulation with IL-4 alone produced no significant effect on IL-8 production. Each column and bar represents mean and standard error.

previous dose dependency studies (data not shown), maximal response was obtained at concentrations greater than $30 \mathrm{ng} / \mathrm{ml}$, consequently these concentrations were used in our study. Recombinant TNF- $\alpha$ (30 ng/ml (3300 $\mathrm{IU} / \mathrm{ml})$ final concentration), IL-4 $(30 \mathrm{ng} / \mathrm{ml}$ (870 IU/ml) final concentration) (purchased from R \& D Systems Inc, Minneapolis, MN, USA) were used for the stimuli. Supernatants were recovered after 24 hours by pipetting and the remaining cells were removed by centrifugation at $800 \mathrm{~g}$ for 5 minutes, and samples were then stored at $-70^{\circ} \mathrm{C}$ until assaying.

CYTOKINE ASSAYS

Levels of immunoreactive human IL-8 and RANTES were quantified using sandwich-type ELISA kits (all purchased from R \& D Systems Inc, Minneapolis, MN, USA) according to the manufacturer's recommended protocol. The limits of detection for IL-8 and RANTES concentrations were $5 \mathrm{pg} / \mathrm{ml}$. Colorimetric results were read at $450 \mathrm{~nm}$ with a microplate reader (MR5000 microplate reader; Dynatech Laboratories, Inc, Chantilly, VA, USA).

Figure 2 RANTES production by corneal epithelial cells (A) and keratocytes (B) ( $n=3$ in triplicate). Cells were stimulated with $T N F-a, I L-4$, and $T N F-a$ and $I L-4$ simultaneously. RANTES was measured in 24 hour cell supernatants by ELISA. In corneal epithelium, simultaneous stimulation with TNF-a and IL-4 produced an increase in RANTES production but was not statistically significant ( $p=0.066$ compared with $T N F-a$ alone). In keratocytes, simultaneous stimulation with $T N F-a$ and IL-4 produced an increase in RANTES production compared with TNF- $\alpha$ induced RANTES production ( $p=0.015$ compared with spontaneous release; $p=0.020$ compared with $T N F-\alpha$ stimulation alone Stimulation with $I L-4$ alone produced no significant effect on RANTES production. Each column and bar represents mean and standard error.

STATISTICAL ANALYSIS

The concentrations obtained by ELISA assays were converted to $\mathrm{ng} / 10^{6}$ cells and were then evaluated using ANOVA test with a post hoc analysis (Fisher PLSD test); $p<0.05$ was considered statistically significant. Statistical analyses were performed using STATVIEW software on a Macintosh computer. All experiments were done in triplicate.

\section{Results}

TNF- $\alpha$ stimulated IL-8 production in both corneal epithelial cells (mean 28 (SE 2.7) $\mathrm{ng} / 10^{6}$ cells, $\mathrm{p}=0.0001$ compared with spontaneous release) and keratocytes (1800 (920) ng $/ 10^{6}$ cells; $\mathrm{p}=0.025$ compared with spontaneous release). Simultaneous addition of IL-4 significantly inhibited the TNF- $\alpha$ induced IL-8 production in both types of cells (corneal epithelial cells; $5.8 \quad(3.8) \mathrm{ng} / 10^{6}$ cells; $\mathrm{p}=0.0002$ : keratocytes; 230 (36) $\mathrm{ng} / 10^{6}$ cells; $\mathrm{p}=0.039)$. All three donors exhibited this pattern in corneal epithelial cells and two of the three donors exhibited this pattern in the case of keratocytes. Stimulation by IL- 4 alone did not induce IL-8 production (Fig 1A, B).

In contrast, RANTES production in corneal epithelial cells increased by simultaneous addi- 
tion of TNF- $\alpha$ and IL- 4 but was not statistically significant $\left(0.36 \quad(0.16) \mathrm{ng} / 10^{6}\right.$ cells; $\mathrm{p}=0.066$ compared with TNF- $\alpha$ alone), two of three donors exhibited this pattern. In keratocytes, simultaneous addition of TNF- $\alpha$ and IL-4 synergistically stimulated RANTES production (9.7 (4.1) $\mathrm{ng} / 10^{6}$ cells; $\mathrm{p}=0.015$ compared with spontaneous release and $\mathrm{p}=0.020$ compared with $\mathrm{TNF}-\alpha$ stimulation alone). All three donors exhibited this pattern. IL-4 alone failed to induce RANTES production in both types of cells (Fig 2A, B).

Keratocytes produced approximately 10 times more IL- 8 and RANTES compared with corneal epithelial cells.

\section{Discussion}

Among cells that are constituents of the ocular surface, it has been reported that the corneal epithelial cells and keratocytes produce IL- $8^{5}$ and that IL- $1 \alpha$ and TNF- $\alpha$ induced RANTES production in keratocytes but not in corneal epithelial cells. ${ }^{7}$ The conjunctival epithelium has also been reported to produce RANTES on exposure to TNF- $\alpha$ and IFN- $\gamma .{ }^{6}$ In our study, we were able to demonstrate that keratocytes and corneal epithelial cells both produced RANTES and IL-8. Tran et al reported that stimulation of keratocytes with TNF- $\alpha$ induced a 200-fold increase in RANTES production. ${ }^{7}$ However, in our study the increase in RANTES production observed was not statistically significant. This is probably due to the fact that in Tran et al's study, the starvation period to exclude the effect of FBS was 3 days compared with 24 hours in our study.

At the same time, we also successfully demonstrated that IL-4 regulates such IL- 8 and RANTES production. In our study, TNF- $\alpha-$ induced IL-8 production was significantly downregulated by simultaneous stimulation with IL-4 in both corneal epithelium and keratocytes. In contrast, TNF- $\alpha$ induced RANTES production was significantly upregulated by simultaneous stimulation with IL-4 in keratocytes. Similar downregulation of IL-8 and upregulation of RANTES by IL- 4 has been observed in monocytes. ${ }^{89}$ As IL-8 is known to be a potent chemoattractant and activator for neutrophils and RANTES, a chemoattractant for eosinophils, ${ }^{3}$ IL-4 downregulation of IL-8 and upregulation of RANTES may result in the decreased infiltration of neutrophils and increased infiltration of eosinophils resulting in the predominance of eosinophils compared with neutrophils in allergic inflammation. This mechanism may play a major part in the selective recruitment of predominantly eosinophils in Th2 dominant situations.

In our study, IL-4 alone failed to induce RANTES and IL- 8 production; these results are consistent with results obtained in previous studies. ${ }^{8} 9$

In a previous study, the mean IL- 4 concentration detected in tears of patients with VKC was $24 \mathrm{pg} / \mathrm{ml}^{4}{ }^{4}$ This concentration is much lower than that used in our study; however, there is a possibility that concentrations of the order used in our study exist at the level of corneal tissue as levels of IL-4 observed in tears are subject to dilution.

Mast cells have been reported to produce TNF- $\alpha$ and IL- 4 on stimulation through their IgE receptors. ${ }^{10}$ Correlation between mast cell activation and eosinophil infiltration has also been reported. ${ }^{11}$ In severe ocular allergies, corneal lesions such as ulcers and erosions are sometimes present, and in such cases the barrier function of the cornea is compromised. This may lead to greater penetration into the corneal tissue of TNF- $\alpha$ and IL- 4 present in the tear fluid. This may result in the upregulation of RANTES and downregulation of IL-8 production by corneal cells as demonstrated in this study.

Furthermore, IL- 4 has also been reported to upregulate the expression of VCAM-1 on vascular endothelium. ${ }^{12}$ As its counterreceptor VLA-4 is expressed on lymphocytes and eosinophils but not on neutrophils; IL-4 may also contribute to the selective recruitment of such cells by this alternative mechanism.

In summary, we demonstrated that corneal epithelial cells and keratocytes produce IL-8 and RANTES. At the same time, we also demonstrated that IL-4 may have a role in the selective recruitment of inflammatory cells to the ocular surface by the differential regulation of chemokine production from corneal cells. Our results may provide a further insight into the mechanisms of corneal damage caused by eosinophils in severe ocular allergies.

The authors thank Miss Midori Hojo for exceptional technical assistance.

1 Trocme SD, Kephart GM, Bourne WM, et al. Eosinophil granule major basic protein deposition in corneal ulcers associated with vernal keratoconjunctivitis. Am $\mathcal{F}$ Ophthalmol 1993;115:640-3.

2 Trocme SD, Hallberg CK, Gill KS, et al. Effects of eosinophil granule proteins on human corneal epithelial cell viability and morphology. Invest Ophthalmol Vis Sci viability and $1997 ; 38: 593-9$.

3 Baggiolini M, Dahinden CA. CC chemokines in allergic Baggiolini M, Dahinden CA. CC chemokines
inflammation. Immunol Today 1994;15:127-33.

4 Fujishima H, Takeuchi T, Shinozaki N, et al. Measurement of IL-4 in tears of patients with seasonal allergic conjunctivitis and vernal keratoconjunctivitis. Clin Exp Immunol 1995;102:395-8.

5 Cubitt CL, Tang Q, Monteiro CA, et al. IL-8 expression in human corneas. Invest Ophthalmol Vis Sci 1993;34:3199206.

6 Fukagawa K, Saito H, Tsubota K, et al. RANTES production in a conjunctival epithelial cell line. Cornea 1997;16: 564-70.

7 Tran MT, Tellaetxe-Isusi M, Elner V, et al. Proinflammatory cytokines induce RANTES and MCP-1 synthesis in human corneal keratocytes but not in corneal epithelial cells. Invest Ophthalmol Vis Sci 1996;37:987-96

8 Standiford TJ, Streiter RM, Chensue SW, et al. IL-4 inhibits the expression of IL-8 from stimulated human monocytes. Immunol 1990;145:1435-9.

9 Marfaing-Koka A, Maravic M, Humbert M, et al. Contrasting effects of IL-4, IL-10 and corticosteroids on RANTES production by human monocytes. Int Immunol 1996;8: 1587-94.

10 Bradding P, Roberts JA, Britten KM, et al. Interleukin-4, -5, and -6 and tumor necrosis factor-alpha in normal and asthmatic airways: evidence for the human mast cell as a source of these cytokines. Am $\mathcal{F}$ Respir Cell Mol Biol 1994;10:471-80

11 Kung TT, Stelts D, Zurcher JA, et al. Mast cells modulate allergic pulmonary eosinophilia in mice. Am F Respir Cell Mol Biol 1995;12:404-9.

12 Gundel R, Lindell D, Harris P, et al. IL-4 induced leucocyte trafficking in cynomolgus monkeys: Correlation with expression of adhesion molecules and chemokine generation. Clin Exp Allergy 1996;26:719-29. 